\title{
Psychometric properties of the Polish version of the eight-item Morisky Medication Adherence Scale in hypertensive adults
}

This article was published in the following Dove Press journal:

Patient Preference and Adherence

9 September 2016

Number of times this article has been viewed

\author{
Beata Jankowska-Polanska' \\ Izabella Uchmanowicz' \\ Anna Chudiak' \\ Krzysztof Dudek ${ }^{2}$ \\ Donald E Morisky ${ }^{3}$ \\ Anna Szymanska- \\ Chabowska $^{4}$ \\ 'Department of Clinical Nursing, \\ Faculty of Health Science, Wroclaw \\ Medical University, Wroclaw, \\ Poland; ${ }^{2}$ Department of Logistic \\ and Transport Systems, Faculty of \\ Mechanical Engineering, Wroclaw \\ University of Technology, Wroclaw, \\ Poland; ${ }^{3}$ Department of Community \\ Health Sciences, UCLA Fielding \\ School of Public Health, Los Angeles, \\ CA, USA; ${ }^{4}$ Department of Internal \\ Medicine, Occupational Diseases \\ and Hypertension, Wroclaw Medical \\ University, Wroclaw, Poland
}

\begin{abstract}
Low adherence to pharmacological treatment is often associated with poor blood pressure control, but identification of nonadherent patients in outpatient settings is difficult. The aim of the study was to translate and evaluate the psychometric properties of the Polish version of the structured self-report eight-item Morisky Medication Adherence Scale (MMAS-8) among patients with hypertension. The study was conducted in a family doctor practice between January and July 2015. After a standard "forward-backward" procedure to translate MMAS-8 into Polish, the questionnaire was administered to 160 patients with hypertension. Reliability was tested using a measure of internal consistency (Cronbach's $\alpha$ ) and test-retest reliability. Validity was confirmed using known group validity. Three levels of adherence were considered based on the following scores: 0 to $<6$ (low); 6 to $<8$ (medium); and 8 (high). Complete questionnaires were returned by 110 respondents (mean age: 60.7 years $\pm 12.6 ; 54.6 \%$ were female). The mean number of pills taken daily was $3.61 \pm 4.31$. The mean adherence score was $6.42 \pm 2.0$. Moderate internal consistency was found (Cronbach's $\alpha=0.81$ ), and test-retest reliability was satisfactory ( $r=0.461-0.905 ; P<0.001)$. Reproducibility expressed by Cohen's $\kappa$ coefficient $=0.61$ was good. In high-adherent patients, the percentage of well-controlled blood pressure was higher than in low-adherent patients ( $33.3 \%$ vs $\left.19.1 \%, \chi^{2}=0.87, P=0.648\right)$. Psychometric evaluation of the Polish version of the MMAS-8 indicates that it is a reliable and valid measure tool to detect nonadherent patients. The MMAS- 8 may be routinely used to support communication about the medication-taking behavior in hypertensive patients.
\end{abstract}

Keywords: Morisky scale, medication adherence, hypertension, validation, pharmacological treatment

\section{Introduction}

Hypertension is one of the most commonly spread risk factors for cardiovascular diseases. The World Health Organization has estimated that one in eight deaths are caused by hypertension; thus, hypertension is considered the third-most common cause of mortality worldwide. ${ }^{1}$ The prevalence of hypertension among adults ranges from $10 \%$ to $20 \%$ and among people over 50 years of age increases up to $40 \%-50 \%$. The prevalence of hypertension in European countries is about $60 \%$ higher than in the USA or Canada. ${ }^{2}$ In Poland, hypertension is diagnosed in $29 \%$ of adult population, and only $12 \%$ of patients are treated properly. ${ }^{3}$

Effective treatment of hypertension is based on lifestyle modification and longterm pharmacotherapy; however, about $40 \%-60 \%$ of patients with hypertension fail to comply with the prescribed treatment ${ }^{4}$ and about $50 \%$ stop taking their drugs during the first 12 months of treatment and $30 \%$ do not take them as prescribed. ${ }^{5}$ Poor blood
Polanska

Department of Clinical Nursing, Faculty of Health Science, Wroclaw Medical University, 5 Bartla Street, Wroclaw $5|-6| 8$, Poland

Tel $+487|784| 824$

Fax +48 7I 3459324

Email bianko@poczta.onet.pl (c)
hereby accept the Terms. Non-commercial uses of the work are permitted without any further permission from Dove Medical Press Limited, provided the work is properly attributed. For permission hereby accept the Terms. Non-commercial uses of the work are permitted without any further permission from Dove Medic
for commercial use of this work, please see paragraphs 4.2 and 5 of our Terms (https://www.dovepress.com/terms.php). 
pressure control is often associated with nonadherence, but on the other hand, poor adherence occurs more often in primary prevention and in asymptomatic patients. ${ }^{4}$

Adherence to antihypertensive medication is a key determinant of adequate blood pressure control and prevention of cardiovascular events, ${ }^{6}$ but studies from the literature show that the recognition of noncompliant patients by health care providers is low $(10 \%-38 \%)$. The lack of blood pressure control may serve as an alarming sign of nonadherence. ${ }^{7}$ Assessment of health records and evaluation of patients' expectations for the treatment may help in identifying nonadherent patients. Also, the important role of questionnaires in the assessment of adherence to therapeutic recommendations is emphasized. ${ }^{8}$

To date, no universal method for the measurement of medication adherence exists, although several scales specific for adherence measurement based on patient's self-assessment are available. Apart from adherence, they often aim at identification of inconvenience related to taking drugs, attitudes and beliefs about treatment, or the level of knowledge about the disease and its treatment. None of the scales are available in the Polish language. ${ }^{9}$

The most commonly used adherence-specific self-report questionnaire is the Morisky Medication Adherence Scale (MMAS). ${ }^{10-12}$ This scale was originally established as a four-item questionnaire in patients with hypertension. It has been expanded with four additional items that address the circumstances regarding adherence behavior. This new version is called the eight-item MMAS (MMAS-8). The ranging score is from 0 to 8 , with higher scores indicating better adherence. The MMAS- 8 has been adapted and validated in many languages and for a variety of chronic diseases that require customized assessment of the recommendations. ${ }^{10}$ Therefore, we adapted it to the Polish culture and carried out its validation in a group of patients with hypertension.

\section{Aim}

In the present study, we report the results of the validation study.

\section{Methods}

\section{Patients and study design}

The study protocol and informed consent form was approved by the independent Commission of Bioethics at Wroclaw Medical University (consent number KB $136 / 2015)$. For the study, patients attending the family doctor practice in Wroclaw between January and July 2015 were enrolled.

\section{Inclusion criteria}

To be included in the study, patients had to:

1. be diagnosed with hypertension at least 1 year before, which is in accordance with the guidelines of the European Society of Hypertension,

2. be treated with antihypertensive medication (at least one drug),

3. be older than 18 years,

4. sign an informed consent, and

5. be able to read Polish.

\section{Exclusion criteria}

The patients excluded from the study:

1. had limited cognitive functions,

2. did not sign an informed consent, and

3. were diagnosed with any other serious disease (neoplasm, cardiac and renal insufficiency, etc).

Two tests were planned in the study at an interval of 4 weeks. At admission, nurses asked the patients to complete the questionnaire. One month after admission, the questionnaire was sent to each patient's home and they were asked to complete it again.

All respondents gave written informed consent for participation in the study. We decided that the best method for standardizing our sample size data was subject to item ratio because we needed a criterion for a reasonable direct comparison to our own data analysis. This corresponds to 20 patients for an eight-item questionnaire. ${ }^{13}$ With this sample size, the width of the $95 \%$ confidence interval for a Cronbach's $\alpha$ coefficient of 0.80 is 0.05 . We decided to include 160 patients.

\section{MMAS questionnaire}

The MMAS-8 is a self-report questionnaire with eight questions. Self-reported medication adherence was assessed using the MMAS-8. The self-reported MMAS-8 tool is simple to administer and is a reliable and an economical method in clinical practice. The MMAS- 8 was designed to facilitate the identification of barriers to and behaviors associated with adherence to chronic medication. The tool has been determined to be reliable and significantly associated with blood pressure control in individuals with hypertension, as well as with antihypertensive medication pharmacy fill rates. Scores on the MMAS- 8 range from 0 to 8 , with scores of $<6$ reflecting low, 6 to $<8$ reflecting medium, and 8 reflecting high adherence. ${ }^{10,14-16}$

\section{Translation and cross-cultural adaptation of the MMAS-8}

The eight items of the MMAS were translated by the Mapi Research Institute according to international guidelines: ${ }^{17,18}$ 
1. Two independent Polish translations were obtained from two independent translators. The Polish version was prepared during a meeting attended by a multidisciplinary team consisting of two physicians, two nurses, and two pharmacists.

2. The reverse translation from Polish to English was carried out by another translator who was not involved in developing the initial version.

3. The original and the back-translated English versions were compared and the problems with inconsistencies resolved in another meeting.

A pilot study was performed in a Polish population of 25 patients. The patients who participated in this face-validity phase were not included further in the study. The final version of the questionnaire was approved by the author of the original version, and then it was completed and made available for the reliability and validity study.

\section{Statistical analysis}

Statistical analysis of the obtained results was carried out with the Statistica software v.10 (StatSoft, Tulsa, OK, USA). Normality of analyzed variables was verified by the Kolmogorov-Smirnov test. Statistical characteristics of variables are presented as arithmetical mean \pm standard deviation (SD), median, and interquartile range. Statistical characteristics of discrete and qualitative variables are presented as frequency distribution (n). Internal consistency and intraclass correlation coefficients of the Polish adaptation of the questionnaire were tested by Cronbach's $\alpha$ coefficient. Exploratory factor analysis was used to identify relationships between questionnaire items. Test-retest reliability was assessed at a 4-week interval to evaluate reproducibility of the Polish version of the questionnaire.

\section{Results}

\section{Clinical and demographic data}

For the study, 160 patients with hypertension who met the inclusion criteria were enrolled. The first study was completed by all of the patients who agreed to participate, while in the repeat study, 43 patients withdrew $(26.8 \%$ refusal rate) and seven returned incomplete questionnaires. The second questionnaire was sent to all the 160 respondents by post, and 43 of them did not send it back. The final analysis included 110 patients with hypertension. The characteristics of the total and adherent groups are shown in Table 1. Of the 110 patients, 27 (24.5\%), 43 (39.1\%), and 40 (36.4\%) were in the low-, medium-, and high-adherence groups, respectively. Significant differences in blood pressure control were found in patients with high adherence who took their medications once or twice a day compared to patients with low adherence who took their medications only when they feel symptoms.

The mean age of respondents was 60.7 years of age (SD, 12.6). Patients in the group with high adherence were older than patients in the group with low adherence (62.9 years, SD 11.0 vs 58.1 years, SD 13.0); however, the difference between those groups was not significant. Of the study group, $54.6 \%$ were women; the percentage of women was higher in the group with high adherence than in the group with low adherence (60\% vs 51.8\%; not significant). Half of the respondents had been educated to a secondary education level. The majority of respondents from the three groups were married or in a relationship (63\% vs $76.7 \%$ vs $60 \%$, respectively). The mean time from diagnosis of hypertension was 9.2 years (SD, 7.4) and was the shortest in the group of patients with high adherence (8.4 years; SD, 4.7). Patients were taking 3.61 (SD, 4.31) antihypertensive tablets a day. Detailed characteristics are presented in Table 1.

Table 2 presents the analysis of adherence levels of MMAS on the group of patients with hypertension. Mean level of adherence according to MMAS-8 was 6.42 (SD, 2.00), which indicates medium adherence to pharmacological recommendation.

\section{Construct validity of the scale}

Two factors were included in the analysis based on the number of plotted points before the last drop. In this way, eight input variables were reduced to two dimensions that explained $59.9 \%$ of the total variance. Factor loading table shows that the most important common factor (Factor 1) is associated mostly with variables describing patients' behavior in the past, while the second one (Factor 2) is associated with taking medicines the day before completing the questionnaire. Factor loadings are presented in Table 3 .

\section{Reliability of the scale}

The Polish version of MMAS-8 adherence scale is a reliable method of verifying medication adherence. The overall Cronbach's $\alpha$ coefficient was 0.808 , and the mean correlation among items of the scale was 0.361 . Table 4 presents basic characteristics of respective items of the scale and their contribution to the whole adherence scale. This table shows that not all items influence the reliability of the scale to a similar extent. The strongest correlation appears between the entire scale and item number 6; thus, the decrease in reliability would also be the strongest, if this was removed from the scale. The weakest influence on the reliability appears in 
Table I Characteristics of patients according to the groups of adherence

\begin{tabular}{|c|c|c|c|c|c|c|}
\hline Characteristic & Total $(n=\mid 10)$ & Low $(n=27)$ & Medium $(n=43)$ & High $(n=40)$ & $\mathbf{F} / \chi^{2}$ & $P$-value \\
\hline Age (y), n (SD) & $60.7(12.6)$ & $58.1(13.0)$ & $60.2(13.6)$ & $62.9(11.0)$ & 1.231 & 0.296 \\
\hline BMI $\left(\mathrm{kg} / \mathrm{m}^{2}\right), \mathrm{n}(\mathrm{SD})$ & $27.8(4.4)$ & $28.9(4.5)$ & $27.6(4.5)$ & $27.3(4.0)$ & 1.081 & 0.343 \\
\hline Female & $60(54.6 \%)$ & 14 (5।.8\%) & $22(51.2 \%)$ & $24(60.0 \%)$ & 0.757 & 0.685 \\
\hline Education level & & & & & 5.271 & 0.261 \\
\hline Primary and below & $3 \mathrm{I}(28.2 \%)$ & $5(18.5 \%)$ & $16(37.2 \%)$ & $10(25.0 \%)$ & & \\
\hline Secondary school & $55(50.0 \%)$ & $17(63.0 \%)$ & $16(37.2 \%)$ & $22(55.0 \%)$ & & \\
\hline College and above & $24(21.8 \%)$ & $5(18.5 \%)$ & II (25.6\%) & $8(20.0 \%)$ & & \\
\hline Marital status & & & & & 3.115 & 0.374 \\
\hline Marriage and partnership & $74(67.3 \%)$ & 17 (63.0\%) & $33(76.7 \%)$ & $24(60.0 \%)$ & & \\
\hline Divorcee/single & 15 (I3.7\%) & $5(18.5 \%)$ & $4(9.3 \%)$ & $6(15.0 \%)$ & & \\
\hline Widow/widower & $21(19.1 \%)$ & $5(18.5 \%)$ & $6(14.0 \%)$ & $10(25.0 \%)$ & & \\
\hline Employment status & & & & & 4.744 & 0.577 \\
\hline Working & $48(43.6 \%)$ & $12(44.4 \%)$ & $20(46.5 \%)$ & $16(40.0 \%)$ & & \\
\hline Retired & $5 \mathrm{I}(46.4 \%)$ & II (40.8\%) & 18 (4I.9\%) & $22(55.0 \%)$ & & \\
\hline Pensioned & $8(7.3 \%)$ & $3(11.1 \%)$ & $3(7.0 \%)$ & $2(5.0 \%)$ & & \\
\hline Unemployed & $3(2.7 \%)$ & I (3.7\%) & $2(4.6 \%)$ & $0(0.0 \%)$ & & \\
\hline Time from HBP diagnosis & $9.5(7.5)$ & $9.3(6.3)$ & $10.9(8.4)$ & $8.2(7.0)$ & 1.343 & 0.265 \\
\hline Classification of hypertension & & & & & 9.283 & 0.158 \\
\hline Normal & $3(2.7 \%)$ & $0(0.0 \%)$ & $0(0.0 \%)$ & $3(7.5 \%)$ & & \\
\hline High-normal & $18(16.4 \%)$ & $4(14.8 \%)$ & $10(23.3 \%)$ & $4(10.0 \%)$ & & \\
\hline Grade I (mild) & $59(53.6 \%)$ & $16(59.3 \%)$ & $20(46.5 \%)$ & $23(57.5 \%)$ & & \\
\hline Grade 2 (moderate) & $30(27.3 \%)$ & $7(25.9 \%)$ & $13(30.2 \%)$ & $10(25.0 \%)$ & & \\
\hline Nicotine addiction & & & & & 7.183 & 0.127 \\
\hline Never smoked & $60(55.0 \%)$ & $12(44.4 \%)$ & $23(53.5 \%)$ & 25 (64.1\%) & & \\
\hline No longer smoker & $20(18.4 \%)$ & $3(11.2 \%)$ & $9(20.9 \%)$ & $8(20.5 \%)$ & & \\
\hline Current smoker & $29(26.6 \%)$ & $12(44.4 \%)$ & II (25.6\%) & $6(15.4 \%)$ & & \\
\hline Alcohol & & & & & 6.636 & 0.465 \\
\hline At all & 37 (33.6\%) & 7 (25.9\%) & $13(30.2 \%)$ & $17(42.5 \%)$ & & \\
\hline Sometimes & $65(59.1 \%)$ & $17(63.0 \%)$ & $26(60.5 \%)$ & $22(55.0 \%)$ & & \\
\hline Often & $7(6.4 \%)$ & $3(11.1 \%)$ & $3(7.0 \%)$ & $\mathrm{I}(2.5 \%)$ & & \\
\hline Daily & $\mathrm{I}(0.9 \%)$ & $0(0.0 \%)$ & I (2.3\%) & $0(0.0 \%)$ & & \\
\hline Frequency of BP control & & & & & 33.46 & 0.001 \\
\hline Three times a day & 9 (8.2\%) & $2(7.4 \%)$ & $3(7.0 \%)$ & $4(10.0 \%)$ & & \\
\hline Twice a day & $32(29.1 \%)$ & 7 (25.9\%) & $10(23.3 \%)$ & $15(37.5 \%)$ & & \\
\hline Once a day & $34(30.9 \%)$ & $5(18.5 \%)$ & $13(30.2 \%)$ & $16(40.0 \%)$ & & \\
\hline Once a week & $14(12.7 \%)$ & I (3.7\%) & $10(23.3 \%)$ & $3(7.5 \%)$ & & \\
\hline Only a doctor & $8(7.3 \%)$ & $3(|\mathrm{I}.| \mathrm{\%})$ & $5(11.6 \%)$ & $0(0.0 \%)$ & & \\
\hline When ill feeling & $13(1 \mathrm{I} .8 \%)$ & $9(33.3 \%)$ & $2(4.6 \%)$ & $2(5.0 \%)$ & & \\
\hline Frequency of medication & & & & & 5.664 & 0.226 \\
\hline More often than once a day & $36(32.7 \%)$ & $10(37.0 \%)$ & 17 (39.5\%) & 9 (22.5\%) & & \\
\hline Once a day & $66(60.0 \%)$ & $14(51.9 \%)$ & $25(58.1 \%)$ & $27(67.5 \%)$ & & \\
\hline Number of medications daily & $3.6 I(4.3 I)$ & $5.42(3.64)$ & $4.75(3.18)$ & $2.64(3.18)$ & 7.862 & 0.062 \\
\hline
\end{tabular}

Note: Statistical significance marked in bold.

Abbreviations: BP, blood pressure; HBP, high blood pressure; BMI, body mass index.

case of item 5 . If item 5 were removed from the scale, the mean correlation between items of the scale would increase to 0.476 . The remaining items would have similar contribution to the reliability, and the reliability coefficient would increase to 0.85 .

\section{Known group validity}

In the group with high adherence, the percentage of patients with properly controlled blood pressure was higher than in the group with low adherence $(33.3 \%$ vs $19.1 \%, P>0.05)$.
Also, mean systolic and diastolic blood pressure were lower in the high-adherent group than in the low-adherent one (141 vs 145 and 84 vs $90 \mathrm{mmHg}$, respectively). Both differences were statistically insignificant $(P>0.05)$. Table 4 shows distribution of levels of adherence in relation to proper blood pressure control.

\section{Test-retest reliability}

A total of 110 questionnaires were returned 1 month after admission and assessed by test-retest method. The results 
Table 2 Analysis of adherence levels of MMAS depending on the degree of illness acceptance

\begin{tabular}{ll}
\hline Morisky Medication Adherence Scale & Moderate illness acceptance (I9-29 points) \\
\hline Do you sometimes forget to take your pills? & $0.66 \pm 0.47$ \\
People sometimes miss taking their medications for reasons other than forgetting. Thinking & $0.81 \pm 0.39$ \\
back over the past 2 weeks, were there any days when you did not take your medicine? & \\
Have you every cut back on or stopped taking your medication without telling your doctor, & $0.83 \pm 0.38$ \\
because you felt worse when you took it? & \\
When you travel or leave home, do you sometimes forget to take your medication with you? & $0.74 \pm 0.44$ \\
Did you take your medicine yesterday? & $0.87 \pm 0.33$ \\
When you feel like your illness is under control, do you sometimes stop taking your medicine? & $0.84 \pm 0.37$ \\
Taking medication every day is a real inconvenience for some people. Do you ever feel hassled & $0.80 \pm 0.40$ \\
about sticking to your treatment plan? & \\
How often do you have difficulty remembering to take all your medications? & $0.88 \pm 0.21$ \\
Never/Rarely (4) & \\
Once in a while (3) & \\
Sometime $(2)$ & \\
Usually $(I)$ & \\
All the time $(0)$ & \\
MMAS-8-Total & $6.42 \pm 2.00$ \\
Adherence level, $n(\%)$ & $27(24.5)$ \\
Low adherence $(<6)$ & $43(39.1)$ \\
Medium adherence $(6-8)$ & $40(36.4)$ \\
High adherence $(=8)$ & \\
\hline
\end{tabular}

Notes: Use of the CMMAS-8 is protected by the US copyright laws. Permission for use is required. A licensure agreement is available from Donald E Morisky, ScD, ScM, MSPH, Professor, Department of Community Health Sciences, UCLA School of Public Health, 650 Charles E Young Drive South, Los Angeles, CA 90095-I772; dmorisky@gmail.com. Data shown as mean \pm SD unless stated otherwise.

Abbreviation: MMAS-8, eight-item Morisky Medication Adherence Scale.

are presented in Table 5 as number of answers, Cohen's $\kappa$ coefficient, Spearman's rank correlation coefficient, and consistency. Correlations between the same items are statistically significant at a level of $P<0.001$, though not equally strong (from $\rho=0.461$ to 0.905 ); the strongest correlation occurred for question $4(\mathrm{Q} 4)$ and the weakest was for question 5 (Q5). The analysis of results obtained during double test among the same people revealed good reproducibility (Cohen's $\kappa$ coefficient $=0.61)$. The overall results are presented in Table 6.

\section{Discussion}

The main objective of this paper was to evaluate the reliability and validity of the translated version of the MMAS- 8 in a sample of patients with hypertension. This study was the first to systematically translate and validate the MMAS- 8 into the Polish language. To date, the MMAS- 8 questionnaire has been translated into several languages and used among patients with hypertension and diabetes..$^{11,17,19-23}$

The evaluation of nonadherence to pharmacological recommendations is necessary in clinical practice, but it still

Table 3 Corrected item-to-total correlation and factors loading of the Polish MMAS-8

\begin{tabular}{|c|c|c|c|c|c|c|}
\hline \multirow[t]{2}{*}{ Items } & \multirow{2}{*}{$\begin{array}{l}\text { Patients' } \\
\text { responses }\end{array}$} & \multirow{2}{*}{$\begin{array}{l}\text { Entry }(n=|| 0), \\
\text { number }(\%)\end{array}$} & \multirow{2}{*}{$\begin{array}{l}\text { Corrected item-to- } \\
\text { total correlation }\end{array}$} & \multirow{2}{*}{$\begin{array}{l}\alpha \text { if } \\
\text { deleted }\end{array}$} & \multicolumn{2}{|l|}{ Factor } \\
\hline & & & & & Factor I & Factor 2 \\
\hline QI & No & $73(66.4)$ & 0.589 & 0.777 & 0.719 & 0.032 \\
\hline Q2 & No & 89 (80.9) & 0.565 & 0.780 & 0.700 & -0.218 \\
\hline Q3 & No & 91 (82.7) & 0.524 & 0.786 & 0.670 & -0.198 \\
\hline Q4 & No & $81(73.6)$ & 0.661 & 0.763 & 0.784 & 0.010 \\
\hline Q5 & Yes & $96(87.3)$ & 0.031 & 0.849 & 0.055 & 0.955 \\
\hline Q6 & No & $92(83.6)$ & 0.686 & 0.762 & 0.800 & -0.003 \\
\hline Q7 & No & $88(80.0)$ & 0.635 & 0.769 & 0.741 & 0.090 \\
\hline \multirow[t]{5}{*}{ Q8 } & Never/rarely & $3(2.7)$ & 0.580 & 0.791 & 0.692 & 0.199 \\
\hline & Once in a while & $10(9.1)$ & & & & \\
\hline & Sometimes & $20(18.2)$ & & & & \\
\hline & Usually & $76(69.1)$ & & & & \\
\hline & All the time & I (0.9) & & & & \\
\hline
\end{tabular}

Note: Statistically significant values are shown in bold.

Abbreviations: $\mathrm{n}$, sample size; MMAS-8, eight-item Morisky Medication Adherence Scale; $\alpha$, Cronbach's alpha coefficient. 
Table 4 Relations between level of adherence and blood pressure control groups

\begin{tabular}{|c|c|c|c|c|}
\hline \multirow{3}{*}{$\begin{array}{l}\text { Adherence level } \\
\text { (MMAS-8) }\end{array}$} & \multicolumn{2}{|c|}{ Blood pressure } & \multicolumn{2}{|l|}{ Mean (SD) } \\
\hline & \multicolumn{2}{|l|}{ Control } & \multicolumn{2}{|c|}{ Blood pressure values } \\
\hline & Yes, n (\%) & No, n (\%) & SBP, $\mathrm{mmHg}$ & DBP, $\mathrm{mmHg}$ \\
\hline Low adherence $(<6)$ & $4(19.1)$ & $23(25.8)$ & $145(17)$ & $90(8)$ \\
\hline Medium adherence $(6$ to $<8)$ & $10(47.6)$ & $33(37.1)$ & $147(14)$ & $91(12)$ \\
\hline High adherence (8) & $7(33.3)$ & $33(37.1)$ & I4I (II) & $84(12)$ \\
\hline
\end{tabular}

Abbreviations: SBP, systolic blood pressure; DBP, diastolic blood pressure; SD, standard deviation; MMAS-8, eight-item Morisky Medication Adherence Scale.

remains difficult. Patient self-assessment related to adherence to prescribed medication seems to be a simple and inexpensive method of obtaining information, as long as properly prepared examination tools are available in daily clinical practice. One of the measures of adherence is the percentage of the medication actually taken by the patient. It must be assumed that the omission of more than $80 \%$ of prescribed doses of medication means total nonadherence, while omission of less than $20 \%$ means partial cooperation. A high percentage of patients admit that they do not take medication daily for various reasons and, moreover, they are not able to accurately calculate the number of omitted doses. ${ }^{7}$

Among the indirect methods of verifying adherence to medication, the following procedures are used the most often: direct observation of treatment process, electronic monitoring systems registering the time of opening canisters with drugs, monitoring of drug concentration in physiological fluids, and patient's response to applied medication with the use of physiological markers such as heart rate. These methods, however, are imprecise and too costly. One of the best assessment methods of the level of adherence is direct interview with a patient including questions about the type of drugs, their doses, and the number of daily doses. Patients without impaired cognitive function and those who cooperate well are able to give correct answers or use a prepared written instruction.

Identification of factors affecting the level of adherence among patients with hypertension is a crucial element in

Table 5 Test-retest result

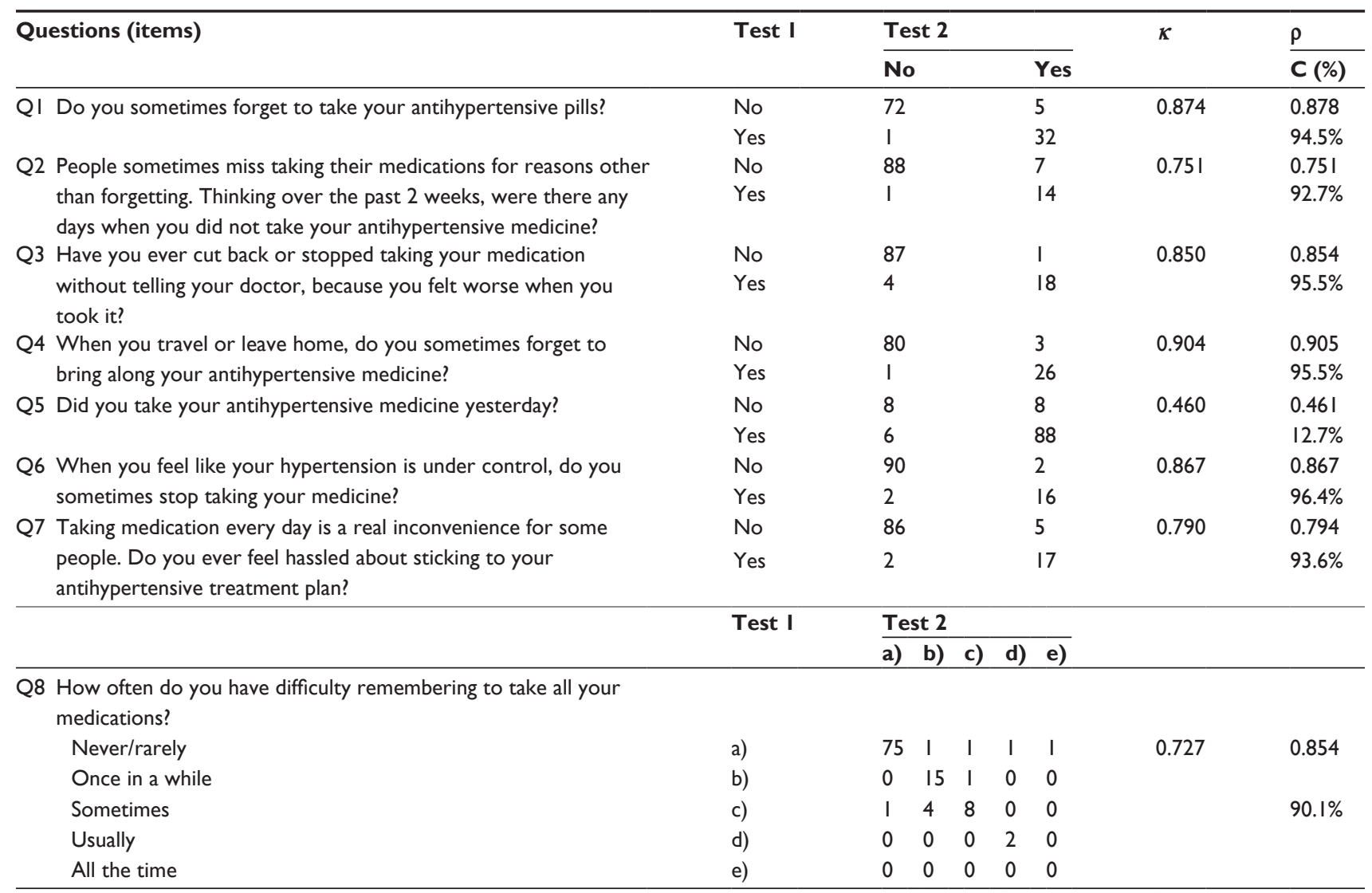

Abbreviations: $\kappa$, Cohen's kappa coefficient; $\rho$, Spearman's rank correlation coefficient; $C$, consistency. 
Table 6 Frequency table and $\chi^{2}$ test

\begin{tabular}{lllll}
\hline $\begin{array}{l}\text { Test } 2 \\
\text { Adherence } \\
\text { level }\end{array}$ & $\begin{array}{l}\text { Test I } \\
\text { Adherence level }\end{array}$ & $\begin{array}{l}\text { Percentage } \\
\text { (\%) }\end{array}$ \\
\cline { 2 - 4 } & Low & Medium & High & \\
\hline Low & 26 & 2 & I & 26.4 \\
Medium & I & 29 & 4 & 30.9 \\
High & 0 & 12 & 35 & 42.7 \\
& $24.5 \%$ & $39.1 \%$ & $36.4 \%$ & \\
\hline
\end{tabular}

Notes: $\chi^{2}=129.6, d f=4, P<0.0001, \kappa=0.606$.

Abbreviations: $d f$, degrees of freedom; $\kappa$, Cohen's kappa coefficient.

improving patients' compliance to following therapeutic recommendations. Based on the available studies, a profile of patients with increased risk for nonadherence can be established. This group includes young men with comorbidities and low socioeconomic status. In addition, it was proved that nonworking, smoking patients with low social support and reduced satisfaction with their medication because of the presence of side effects of the treatment have significantly greater amount of problems with adherence to medication regimens. ${ }^{21}$

The original MMAS-8 scale was tested by Morisky et $\mathrm{al}^{10}$ on a sample of hypertensive patients, and it was found that the scale was reliable with good predictive validity and sensitivity (Cronbach's $\alpha=0.83$ ) and was significantly associated with blood pressure control $(P<0.05)$. Other studies evaluated the MMAS- 8 translated version in Brazilian Portuguese, Thai, French, Malaysian, Korean, Persian, and Chinese. ${ }^{11,19-21,23-25}$ These studies show that the MMAS-8 translated version had acceptable internal consistency, good test-retest reliability, and good convergent validity. The questionnaire was primarily dedicated to measure adherence among patients with hypertension; however, currently, it is also often used among diabetic and cardiac patients as well as in patients with asthma, psoriasis and AIDS. ${ }^{20,22,24,26}$

The MMAS-8 questionnaire was translated into several languages. The level of internal consistency of Polish adaptation of MMAS- 8 was $\alpha=0.81$, and the mean correlation between items of the scale was 0.36 . The values are similar to those obtained for the original version of the questionnaire. ${ }^{10}$ The psychometric properties of the foreign versions were recognized as satisfactory, although their parameters of internal consistency were lower than those for the Polish version. The Cronbach's $\alpha$ was 0.682 for the Brazilian Portuguese version, 0.54 for the French version, 0.697 for the Persian version, while it was 0.808 for the Polish version. ${ }^{11,12,21}$

Our study among Polish patients with hypertension showed that the MMAS-8 had good test-retest reliability $(r=0.61)$ with good convergent validity and sensitivity
(59.9\%). In terms of internal consistency and test-retest reliability, it is different from the results of de OliveiraFilho et $\mathrm{al}^{11}$ and Moharamzad et $\mathrm{al}^{12}$. These authors, however, performed the reproducibility assessment after 2 weeks, which showed that the ability to change adherence to pharmacological recommendations is less possible in such a short period of time - in the present study, the interval between questionnaires was 4 weeks. The Polish version of MMAS-8 questionnaire was able to differentiate significantly between patients with well-controlled and uncontrolled blood pressure. In the present study, patients with well-controlled blood pressure obtained higher score in MMAS-8 questionnaire, showing better adherence than those with uncontrolled blood pressure. Significant correlation between MMAS-8 questionnaire and the level of blood pressure control was confirmed in the study on the original version of the questionnaire and in the studies of other researchers. ${ }^{10,11}$

\section{Conclusion}

Identification of nonadherent patients is important to improve the effectiveness of antihypertensive treatment. The Polish version of MMAS- 8 questionnaire is a reliable and valid measure of medication adherence in hypertensive patients. This questionnaire should be used in daily clinical practice, especially in patients with uncontrolled hypertension despite correctly applied therapeutic regimens. Recognition of nonadherent patients along with finding reasons for their lack of adherence may allow for the development of strategies for improving adherence. The Polish version of the questionnaire may be used in routine care to support communication about the medication-taking behavior.

\section{Acknowledgments}

The authors thank Professor Dariusz Janczak for his assistance in preparing the paper and Dr Mariusz Chabowski for his help with writing the article.

\section{Disclosure}

Donald E Morisky receives honorarium from use of the MMAS scales. The authors report no other conflicts of interest in this work.

\section{References}

1. Kearney PM, Whelton M, Reynolds K, Muntner P, Whelton PK, He J Global burden of hypertension: analysis of worldwide data. Lancet. 2005;365:217-223.

2. Wolf-Maier K, Cooper RS, Banegas JR, et al. Hypertension prevalence and blood pressure levels in 6 European countries, Canada, and the United States. JAMA. 2003;289:2363-2369. 
3. Kawecka-Jaszcz K, Pośnik-Urbańska A, Jankowski P. [Prevalence of arterial hypertension in Poland - impact of gender]. Nad Tęt. 2007;11(5): 377-383. Polish.

4. Marcum ZA, Zheng Y, Perera S, et al. Prevalence and correlates of self-reported medication non-adherence among older adults with coronary heart disease, diabetes mellitus, and/or hypertension. Res Social Adm Pharm. 2013;9:817-827.

5. Karakurt P, Kasikci M. Factors affecting medication adherence in patients with hypertension. J Vasc Nurs. 2012;30:118-126.

6. Hedegaard U, Kjeldsen LJ, Pottegard A, et al. Improving medication adherence in patients with hypertension: a randomized trial. Am J Med. 2015;128(12):1351-1361.

7. Christensen A, Osterberg LG, Hansen EH. Electronic monitoring of patient adherence to oral antihypertensive medical treatment: a systematic review. J Hypertens. 2009;27:1540-1551.

8. Perez-Escamilla B, Franco-Trigo L, Moullin JC, Martinez-Martinez F, Garcia-Corpas JP. Identification of validated questionnaires to measure adherence to pharmacological antihypertensive treatments. Patient Prefer Adherence. 2015;9:569-578.

9. Klocek M. Współpraca chorego z lekarzem i przestrzeganie zaleceń terapeutycznych [The cooperation of the patient and physician adherence to treatment]. In: Więcek A, Januszewicz A, Szczepańska-Sadowska E, Narkiewicz K, Prejbisz K, Cudnoch-Jędrzejewska A, editors. Hypertensjologia. Patogeneza, diagnostyka i leczenia nadciśnienia tętniczego. [Hypertensiology. The pathogenesis, diagnosis and treatment of hypertension]. Kraków: Medycyna Praktyczna [Practical Medicine], 2015:477-481. Polish.

10. Morisky DE, Ang A, Krousel-Wood M, Ward HJ. Predictive validity of a medication adherence measure in an outpatient setting. $J$ Clin Hypertens (Greenwich). 2008;10:348-354.

11. de Oliveira-Filho AD, Morisky DE, Neves SJ, Costa FA, de Lyra DP Jr. The 8-item Morisky Medication Adherence Scale: validation of a Brazilian-Portuguese version in hypertensive adults. Res Social Adm Pharm. 2014;10:554-561.

12. Moharamzad Y, Saadat H, Nakhjavan SB, et al. Validation of the Persian Version of the 8-Item Morisky Medication Adherence Scale (MMAS-8) in Iranian hypertensive patients. Glob J Health Sci. 2015;7:173-183.

13. Costello A, Osborne JW. Best practices in exploratory factor analysis: four recommendations for getting the most from your analysis. Pract Assess Res Eval. 2005;10(7):1-9.

14. Holt EW, Munter P, Joyce C, Morisky DE, Webber LS, Krousel-Wood M. Life events, coping, and antihypertensive medication adherence among older adults. Am J Epidemiol. 2012;176(Suppl):S64-S71.
15. Krousel-Wood M, Islam T, Webber LS, Re RN, Morisky DE, Muntner P. New medication adherence scale versus pharmacy fill rates in seniors with hypertension. Am J Manag Care. 2009;15(1):59-66.

16. Morisky DE, DiMatteo MR. Improving the measurement of selfreported medication nonadherence: final response. J Clin Epidemio. 2011;64:258-263.

17. Wild D, Grove A, Martin M, et al. Principles of good practice for the translation and cultural adaptation process for patient-reported outcomes (PRO) measures: report of the ISPOR task force for translation and cultural adaptation. Value Health. 2005;8:94-104.

18. Beaton DE, Bombardier C, Guillemin F, Ferraz MB. Guidelines for the process of cross-cultural adaptation of self-report measures. Spine (Phila Pa 1976). 2000;25:3186-3191.

19. Al-Qazaz HK, Hassali MA, Shafie AA, Sulaiman SA, Sudram S, Morisky DE. The eight-item Morisky Medication Adherence Scale MMAS: translation and validation of the Malaysian version. Diabetes Res Clin Pract. 2010;90:216-221.

20. Lee WY, Ahn J, Kim JH, et al. Reliability and validity of a self-reported measure of medication adherence in patients with type 2 diabetes mellitus in Korea. J Int Med Res. 2013;41:1098-1110.

21. Korb-Savoldelli V, Gillaizeau F, Pouchot J, et al. Validation of a French version of the 8-item Morisky medication adherence scale in hypertensive adults. J Clin Hypertens (Greenwich). 2012;14:429-434.

22. Sakthong P, Chabunthom R, Charoenvisuthiwongs R. Psychometric properties of the Thai version of the 8-item Morisky Medication Adherence Scale in patients with type 2 diabetes. Ann Pharmacother. 2009; 43:950-957.

23. Culig J, Leppee M. From Morisky to Hill-bone; self-reports scales for measuring adherence to medication. Coll Antropol. 2014;38:55-62.

24. Wang J, Bian R-W, Mo Y-Z. Validation of the Chinese version of the eight-item Morisky medication adherence scale in patients with type 2 diabetes mellitus. J Clin Gerontol Geriatr. 2013;4:119-122.

25. Arnet I, Metaxas C, Walter PN, Morisky DE, Hersberger KE. The 8-item Morisky Medication Adherence Scale translated in German and validated against objective and subjective polypharmacy adherence measures in cardiovascular patients. J Eval Clin Pract. 2015;21:271-277.

26. Oguzulgen IK, Kokturk N, Isikdogan Z. [Turkish validation study of Morisky 8-item medication adherence questionnaire (MMAS-8) in patients with asthma and chronic obstructive pulmonary disease]. Tuberk Toraks. 2014;62:101-107. Turkish.
Patient Preference and Adherence

\section{Publish your work in this journal}

Patient Preference and Adherence is an international, peer-reviewed, open access journal that focuses on the growing importance of patient preference and adherence throughout the therapeutic continuum. Patient satisfaction, acceptability, quality of life, compliance, persistence and their role in developing new therapeutic modalities and compounds to optimize

\section{Dovepress}

clinical outcomes for existing disease states are major areas of interest for the journal. This journal has been accepted for indexing on PubMed Central. The manuscript management system is completely online and includes a very quick and fair peer-review system, which is all easy to use. Visit http://www. dovepress.com/testimonials.php to read real quotes from published authors. 